\title{
Towards an Ontology to Support Decision-making in Hospital Bed Allocation (TSE)
}

\author{
Debora Engelmann, Julia Couto, Vagner Gabriel, Renata Vieira, and Rafael H. Bordini \\ School of Technology, PUCRS - Pontifical Catholic University of Rio Grande do Sul - Porto Alegre, Brazil \\ Email: [debora.engelmann, julia.couto, vagner.gabriel]@edu.pucrs.br, [renata.vieira, rafael.bordini]@pucrs.br
}

\begin{abstract}
Using advanced technologies is imperative to support quick decision-making in a hospital, where people work with complex and critical processes. An example of a complex and very important task is to make the best decision about in which room and bed a patient should be admitted to hospital, considering the patient needs, characteristics, and available resources. In this case, bad decisions can even compromise patient health. With this study, we aim to facilitate patient-related decisions related to bed allocation, based on an ontology. To do so, we developed an ontology that takes patients' information into account to help health professionals decide where to allocate them. Our main contribution is an ontology, with classes, relationships, individuals, and rules to be used in specific scenarios. Additionally, we exemplify some scenarios in which the rules could be applied.

Index Terms-Ontology, health care, hospital bed allocation.
\end{abstract}

\section{INTRODUCTION}

Hospital decision-making is an important and complex task, which demands a great deal of cognitive effort from health professionals [13]. In this way, tools and technologies that facilitate decision making are great alternatives to help health professionals reduce their cognitive load and possible errors caused by fatigue.

Information technology is widely adopted in modern medical practice, especially to support administrative tasks, patient electronic records, and data management [15]. Artificial Intelligence (AI) has also been highlighted in several applications in the medical field, e.g. to predict patient inflow [8], identify high-quality physicians based on big data [24], and estimate surgical time duration [21].

Ontologies provide the basis to establish an explicit formal concept specification in a specific domain, allowing the development of relationships between these concepts and the reuse and integration of domain knowledge [10]. In the hospital domain, ontologies provide information associated with a wealth of knowledge about clinical decisions [3], medication [2], diagnoses [19], medical records [11], and so on.

In this paper, we report the development of an ontology for bed allocation, which we developed for the Portuguese language. Our ontology is composed of classes, object properties, and individuals, enabling us to present real scenarios that can be tested using its rules. The main function of our ontology is to assist decision making about the place where patients will be allocated when being admitted to hospital, according to the patient's records, characteristics, and the bed allocation rules used in the particular hospital.

DOI reference number: 10.18293/SEKE2019-130

\section{TheOretiCAL BACKGROUND}

The development, dissemination, and use of common communication standards, vocabularies, and ontologies are important for the development of health systems [13]. Therefore, in this section we explore some of the main concepts related to our work, such as hospital bed allocation and ontologies.

\section{A. Hospital bed allocation}

Hospital bed management is an important part of operational capacity planning and control, and it involves the efficient use of resources [17]. Each hospital has its own rules, but there are some factors that must be considered when choosing a bed for a patient, such as age, patient condition, and gender.

Effective management of beds has always been a challenge for managers, given that hospital settings are highly dynamic and uncertain. It is necessary to accommodate both scheduled and emergency patients, requiring multiple expertise in a wide range of hospital departments with various different constraints [4].

Furthermore, in a hospital there are many other resources that need to be allocated and many decisions that always need to be made as quickly as possible. An ontology-based decision support system can be useful to quickly make the right decisions, even helping save lives.

\section{B. Ontology}

An ontology is an explicit and formal specification of a shared conceptualisation made up of concepts or classes, relationships, instances, attributes, axioms, restrictions, rules, and events [20]. An ontology communicates what kinds of things exist and how they are related to each other [22]. A standard for representing ontologies that is widely used both in academia and industry is the OWL (Ontology Web Language), is a language for representing ontologies that is based on formal logic, a discipline that evolved from philosophy and mathematics [22].

For instance, Protég $\oint^{1}$ is a open source tool that implements OWL. A Protégé ontology consists of classes, properties, individuals, and rules. Classes are concepts in the domain of discourse. Object properties are slots that describe properties or attributes of classes. Individuals are class instances, and rules are axioms specifying additional constraints. The ontology and its class individual instances with specific values comprise a knowledge base on Protégé [14].

\footnotetext{
${ }_{1}^{1}$ https://protege.stanford.edu
} 


\section{Bed Allocation Ontology}

The inspiring approach [5] was used in the development of the ontology containing 95 classes, 85 object properties, and 78 individuals. We created the original version in Portuguese, but we translated the terms here for consistency with the study report. Next, we explain its components, and present and exemplify the rules we created based on the hospital bed management context. Due to space constraints, we are not able to present here all the details, properties, rules and ontology visual representation, so we provide it in a repository at GitHub: https://github.com/DeboraEngelmann/Hospital-BedAllocation-Ontology.

\section{A. Classes}

In this section, we detail the classes we created. Note that all the concepts described here refer to the scenario created by the researchers, and they can have different meanings in different contexts.

Attendance: The term attendance is used to refer to the whole period the patient attended any activity in a hospital, either ambulatorial or hospitalisation. Ambulatorial is the assistance that occurs via prior scheduling or by emergency need. It has Elective (when it is scheduled) and Emergency sub-classes (when it occurs without scheduling, because of an urgent need of the patient). Hospitalisation occurs when the patient needs to stay in hospital for more than one day, occupying a Hospital_Bed.

Risk_Classification: A risk category assigned to patients when they start being cared for, widely used in the emergency sector to prioritise patients in worst health conditions. We describe it based on Manchester protocol, defined by Mackway-Jones, Marsden, and Windle [9]. Sub-classes range from immediate to non urgent. A patient that has risk of death is classified as Very_urgent. Patients with immediate risk of limb loss or loss of organ function are classified as Very_urgent. Patients with conditions that can worsen if not helped soon are Urgent. Patients with low risk of health damage are Standard, and the ones without any immediate risk of health damage are Non_urgent.

Temporal_concept: Concepts related to the timing of events. It includes the following sub-classes: Now, Year, Date, Day, Today, Hour, Time interval, Month, and Week.

Document: It refers to the documents generated during or after a patient's attendance. It includes diagnosis, report, prescription, and medical records. (1) Diagnosis: made by a doctor, it determines the disease nature and cause, based on the patient history, symptoms, examination, etc. (2) Report: made by a specialist doctor, it usually contains the analysis of exams, such as radiology, laboratory, etc. (3) Prescription: made by a doctor, it includes drugs and treatments recommended to the patient. 4) Medical_records: it includes all the data related to the patient that can be accessed and stored by the hospital.

Disease: Biological alteration of a person's health state, manifested by a set of symptoms.

Speciality: Represents the medical specialisation or expertise that the doctor possesses or that the patient needs.
State: it represents patient conditions, and has 5 sub-classes: Coma, In treatment, Stable, Severe, and Vegetative.

Situation: it represents hospital bed conditions, and has 5 sub-classes: Blocked, Clean, Free, Occupied, and Dirty.

Local: places inside a hospital. Sub-classes are: Corridor, Pharmacy, Bed, Room, Reception, and Hospitalisation_Unit. The hospitalisation_Unit also has sub-classes named: Speciality_Unit, Nursery, Pediatrics, Intensive_Care Unit, and Special_Care_Unit.

Medication: the drugs stored on the Pharmacy, which are meant to treat the patients.

Furniture: All the furniture that belongs to the hospital. For this study, we describe just two sub-classes: Bed and Stretcher.

Person: People who belong to the hospital ecosystem. Sub-classes are: Companion, Man, Woman, Patient, and Employee. Employee has the following sub-classes: Administration, Cleaner, Receptionist, Security_Guard, and Health_Professional. The last one comprehends Nurse, Nursing_Technician, and Doctor. Doctor also have sub-classes named: Generalist, Resident, and Specialist. Specialist has the following sub-classes: Cardiologist, Dermatologist, Neurologist, Oncologist, Pediatrician, Pneumologist, Radiologist, and Traumatologist.

Restriction: rules to restrict bed allocation. (1) Routing: Origin of the patient, for example if he came from the emergency or is an elective patient. (2) Age: person's age group, which can be adult, teenager, or child. (3) Gender: male or female. (4) Isolation: Refers to the cases where the patient cannot be in the a room with other patients. (5) Puerperal: Women who just gave birth. (6) Length_Of_Stay: Predicted time of patient stay in the hospital, can be turn-fast or longstay. (7) Hospital_Care: Hospital care the patient needs, can be surgical or clinical. (8) Type_Of_Care: Type of care that the patient needs, can be minimal, semi-intensive or intensive.

Symptom: Signs to which the patient refers when talking about his illness (pain, fever, etc.).

Treatment: Set of instructions of procedures that the doctor recommends for the patient undergo.

\section{B. Object Properties}

We created 85 relationships between the classes, and some of them presented in Table II The full list is available at GitHub. We have not included all possible relationships between classes, only those we consider interesting for this domain, so we could see clearly how the classes relate to each other, to help us test the rules.

\section{Individuals}

We instantiated 78 individuals, so we could use the reasoner to test our rules. Individuals we created include Patient, Room, Hospital_Bed, Symptoms, and so on. To create them, we used names such as Patient1, 100,100A, Headache, and so forth.

\section{Rules}

Our ontology aims to help decision making about the beds where patients can be allocated according to the bed 
TABLE I

Bed Allocation Ontology - Object Properties

\begin{tabular}{|llll|}
\hline Domain & Object Property & Range & Inverse of \\
\hline Attendance & happens-in & Temporal_concept & by \\
Nurse & allocates & Hospital_Bed & is-allocated-by \\
$\begin{array}{l}\text { Health_Profess. } \\
\text { Patient } \\
\text { Employsee }\end{array}$ & $\begin{array}{l}\text { presents-one } \\
\text { attend }\end{array}$ & $\begin{array}{l}\text { Document } \\
\text { Disease }\end{array}$ & is-analysed-by \\
Doctor & $\begin{array}{l}\text { Patient } \\
\text { attend-the- }\end{array}$ & Speciality & is-attended-by \\
$\begin{array}{l}\text { Health_Profes. } \\
\text { Patient }\end{array}$ & evaluates & Patient & is-evaluated-by \\
consumes & Medication & is-consumed-by \\
Doctor & discharges & Patient & is-discharged-by- \\
Patient & vacates-one & Hospital_Bed & is-vacated-by \\
Doctor & diagnoses-one & Patient & is-diagnosed-by \\
& & & \\
\hline
\end{tabular}

allocation constraints. Thus, we establish rules that propagate information about restrictions by registered individuals. We are aware that a lot of rules can be created to help decision making related to bed allocation in hospitals. We present a sample of the ones we created for our ontology in Table II.

1) First scenario: For some hospitals, there are a number of bedrooms that have no preferential gender. It means one can allocate male or female patients to those bedrooms, but not both.Let us say there is a bedroom with two empty beds, and then a man is allocated to one of those beds. Thereafter, while there is a man in this bedroom, only another man can occupy the other bed. Nevertheless, as soon as the bedroom is empty, women can occupy it too, according to the priority in the queue of patients to be hospitalised. In this scenario, our rules work as follows. If a male patient is placed in a bed, RULE 1 infers that the patient is male, RULE 23 infers that the bed has become male only, RULE 29 infers that if a bed of the bedroom is for male patients, then the bedroom must also be male only, and finally, RULE 19 infers that if a bedroom is male only, all the beds in that room are too.

2) Second scenario: Patient disease and condition also influences bed allocation. For instance, when a woman is suspected to have a disease transmitted by air and highly contaminating, she must be isolated, having her own bedroom, and be attended by a Pneumologist doctor. In this case, when allocating this patient in a bed, RULE 21 infers that if the patient is of the Pneumology speciality then the bed where she was allocated is also of that speciality. RULE 9 infers that the bedroom where this bed is also is of the Pulmonology speciality. Finally, RULE 8 infers that if there are other beds in that bedroom, they all have that same speciality. In addition, because it is a patient in isolation, rule 20 infers that if the patient is in isolation, so is the bed. RULE 2 infers that if the bed is for isolation then the bedroom should also be. And

\begin{tabular}{|c|c|c|c|}
\hline Domain & Object Property & Range & Inverse of \\
\hline Hospital_Bed & $\begin{array}{l}\text { bed-is-of-the- } \\
\text { age-group }\end{array}$ & Age & \\
\hline Hospital_Bed & $\begin{array}{l}\text { bed-is-the- } \\
\text { attendance }\end{array}$ & Hospital_Care & \\
\hline Hospital_Bed & bed-is-care & Type_Of_Care & \\
\hline Hospital_Bed & bed-is-routing & Routing & \\
\hline Hospital_Bed & bed-is-stay & Length_Of_Stay & \\
\hline Hospital_Bed & $\begin{array}{l}\text { bed-is-of-the- } \\
\text { gender }\end{array}$ & Gender & \\
\hline Hospital_Bed & $\begin{array}{l}\text { bed-is- } \\
\text { puerperal }\end{array}$ & Puerperal & \\
\hline Hospital_Bed & own-one & Situation & \\
\hline Bedroom & $\begin{array}{l}\text { bedroom-is- } \\
\text { speciality }\end{array}$ & Speciality & \\
\hline Bedroom & $\begin{array}{l}\text { bedroom-is- } \\
\text { the-attendance }\end{array}$ & Hospital_Care & \\
\hline Bedroom & $\begin{array}{l}\text { bedroom-is- } \\
\text { care }\end{array}$ & Type_Of_Care & \\
\hline Bedroom & $\begin{array}{l}\text { bedroom-is- } \\
\text { routing }\end{array}$ & Routing & \\
\hline
\end{tabular}

finally, RULE 27 infers that all beds in the bedroom should be of the same type of isolation.

3) Third scenario: Another important restriction related to bed allocation is age group. When a child has to be hospitalised, we can only allocate them in the same bedroom with people up to 12 years old. The rules we can apply in this case are: RULE 24 that infers that if the patient in a bed is of a certain age group then the bed is also; RULE 16 infers that the bedroom is the same age group as the bed that has a person allocated; and RULE 18 that infers that all other beds in the bedroom have the same age group.

4) Fourth scenario: When a healthy baby is born, its mother must be allocated in a bed in the Maternity Unit, because she needs a baby crib next to her bed. In this case, RULE 12 infers that if the patient is of a puerperal type, then the bed is of the same type. RULE 7 infers that the bedroom to which the bed belongs will be of the same puerperal type, and RULE 28 infers that in this case, the other beds in that bedroom must only be allocated women who just gave birth to a healthy child. In addition to these scenarios, we create rules with this same type of reasoning for all other constraints.

\section{RELATED WORK}

A considerable number of papers studied ontologies on hospital domains. In this Section, we summarise some of them. Rubin [18] presents Radlex, a standard terminology widely used in the radiology field. Mhiri and Despres [12] use ontologies as indexing support to generate more accurate clinical reports. Kataria et al. developed HIWO [6], a formal description of an intelligent hospital domain, based on a ontology. The Nurse Call System (oCS) [15], developed by Ongenae et al., uses an ontology to manage context information about patient profiles and team members. An Ontology for Hospital Scenarios (OntHoS), created by Becker et al. [1], aims to establish a basis for a description of these 
TABLE II

RULES OF THE ONTOLOGY

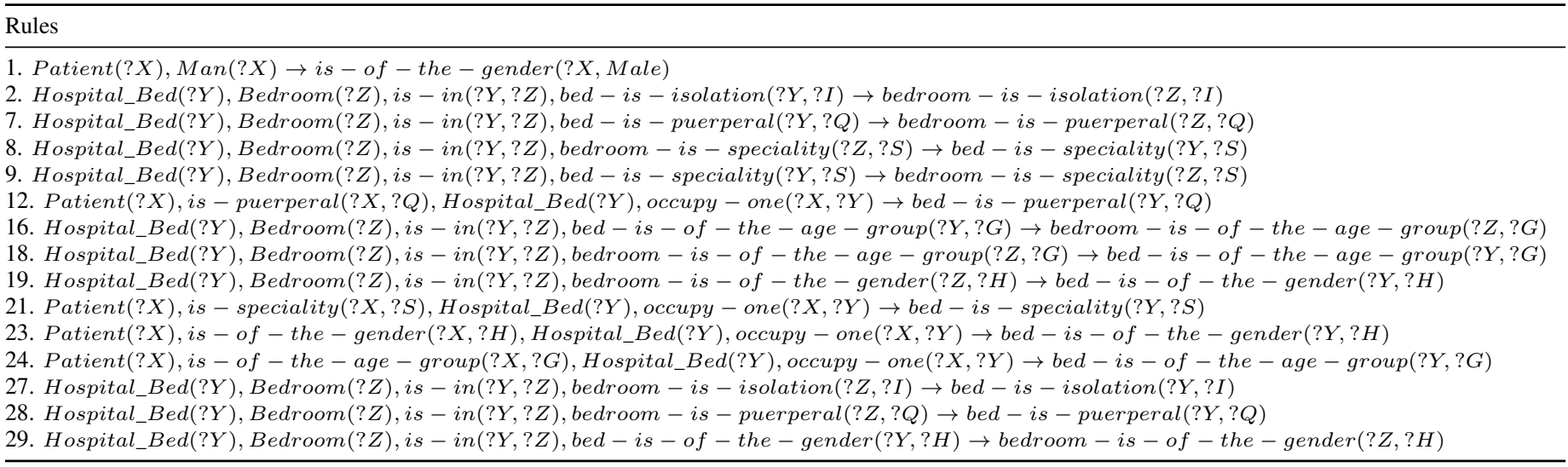

scenarios and facilitate their interoperability. We also found a UML-based ontology to describe hospital information system architectures [23], an ontology for intelligent assistants in patient management based on rules [16], and an ontology-based health context information model to implement omnipresent environments [7]. Different from our work, none of them focuses on bed allocation.

\section{CONCLUSIONS}

In this paper we presented the concepts, properties, and rules we developed for an ontology that can be used to help decision making about the place where the patients will be allocated when hospitalised, according to predefined bed allocation rules. We also present four different examples of scenarios in which the ontology could be used. Our ontology has not been used yet in a real scenario, although it is freely available online and we hope it can motivate people to use it. The full version of our ontology has 95 classes, 85 object properties, and 78 individuals. As future work we plan to evaluate our ontology with domain specialists. Our ontology can also be extended and explored in other directions such as the definition of the number of patients per nurse, distribution and classification of the patients in beds, among other possibilities.

\section{REFERENCES}

[1] M. Becker et al., OntHoS - an Ontology for Hospital Scenarios. Birkhäuser Verlag, 2003, pp. 87-103.

[2] J.-J. Chen et al., "Applying ontology techniques to develop a medication history search and alert system in department of nuclear medicine," Journal of Medical Systems, vol. 36, no. 2, pp. 737-746, Apr 2012.

[3] B. Cánovas-Segura et al., "A lightweight acquisition of expert rules for interoperable clinical decision support systems," Knowledge-Based Systems, vol. 167, pp. 98 - 113, 2019.

[4] M. d. S. Grübler et al., "A hospital bed allocation hybrid model based on situation awareness," CIN, vol. 36, no. 5, pp. 249-255, May 2018.

[5] C. W. Holsapple et al., "A collaborative approach to ontology design," Commun. ACM, vol. 45, no. 2, pp. 42-47, Feb 2002.

[6] P. Kataria et al., "Implementation of ontology for intelligent hospital wards." in HICSS. IEEE Computer Society, 2008, p. 253.

[7] J. Kim et al., "Ontology-based healthcare context information model to implement ubiquitous environment," Multimedia Tools and Applications, vol. 71, no. 2, pp. 873-888, Jul 2014.
[8] D. A. Kottalanka Srikanth, "An efficient patient inflow prediction model for hospital resource management," Indonesian Journal of Electrical Engineering and Computer Science, vol. 7, no. 3, pp. 809-817, 2017.

[9] K. Mackway-Jones et al., Emergency triage: Manchester triage group. John Wiley \& Sons, 2013.

[10] H. Martin et al., "Medical ontologies for machine learning and decision support," mar 2011, uS Patent 7,899,764.

[11] R. Messaoudi et al., "An ontological model for analyzing liver cancer medical reports," in Information Systems, M. Themistocleous et al., Eds. Cham: Springer International Publishing, 2019, pp. 369-382.

[12] S. Mhiri et al., "Ontology usability via a visualization tool for the semantic indexing of medical reports (dicom sr)," in USAB. Springer, 2007, pp. 409-414.

[13] J. Nealon et al., "Agent-based applications in health care," in Applications of software agent technology in the health care domain. Springer, 2003, pp. 3-18.

[14] N. F. Noy et al., "The knowledge model of protégé-2000: Combining interoperability and flexibility," in Knowledge Engineering and Knowledge Management Methods, Models, and Tools, R. Dieng et al., Eds. Berlin, Heidelberg: Springer Berlin Heidelberg, 2000, pp. 17-32.

[15] F. Ongenae et al., "An ontology-based nurse call management system (oncs) with probabilistic priority assessment," BMC Health Services Research, vol. 11, no. 1, p. 26, Feb. 2011.

[16] V. L. Payne et al., "Hospital care watch (hcw): an ontology and rulebased intelligent patient management assistant," in $C B M S$, June 2005, pp. $479-484$.

[17] N. C. Proudlove et al., "Can good bed management solve the overcrowding in accident and emergency departments?" Emergency Medicine Journal, vol. 20, no. 2, pp. 149-155, 2003.

[18] D. L. Rubin, "Creating and curating a terminology for radiology: ontology modeling and analysis," Journal of digital imaging, vol. 21, no. 4, pp. 355-362, 2008.

[19] L. Subirats et al., Personalization of Ontologies Visualization: Use Case of Diabetes. Cham: Springer International Publishing, 2019, pp. 3-24.

[20] M. Tovar et al., "A metric for the evaluation of restricted domain ontologies," Computación y Sistemas, vol. 22, no. 1, pp. 147-162, Mar 2018.

[21] J. P. Tuwatananurak et al., "Machine learning can improve estimation of surgical case duration: A pilot study," Journal of Medical Systems, vol. 43, no. 3, p. 44, Jan 2019.

[22] M. Uschold, "Demystifying owl for the enterprise," Synthesis Lectures on Semantic Web: Theory and Technology, vol. 8, no. 1, pp. i-237, May 2018.

[23] A. Winter et al., "A uml-based ontology for describing hospital information system architectures," Studies in health technology and informatics, vol. 84, no. Pt 1, p. 778-782, 2001.

[24] Y. Ye et al., "A hybrid it framework for identifying high-quality physicians using big data analytics," International Journal of Information Management, vol. 47, pp. 65 - 75, 2019. 